Article

\title{
Acute Effects of Whole-Body Vibration Exercise on Pain Level, Functionality, and Rating of Exertion of Elderly Obese Knee Osteoarthritis Individuals: A Randomized Study
}

\author{
Marcia Cristina Moura-Fernandes ${ }^{1,2}$, Eloá Moreira-Marconi 1,2 ${ }^{\mathbb{D}}$, \\ Alexandre Gonçalves de Meirelles ${ }^{2,3}$, Aline Reis-Silva 2,3, Luiz Felipe Ferreira de Souza ${ }^{2}$ (D), \\ Adriana Lírio Pereira da Silva ${ }^{2,4}$, Bruno Bessa Monteiro de Oliveira ${ }^{1,2,5}$ (D), \\ Marco Antonio de Souza Gama ${ }^{2,5}$, Ana Carolina Coelho de Oliveira ${ }^{1,2,6}$, \\ Daniel Batouli-Santos ${ }^{2}$, Ygor Teixeira da Silva ${ }^{2,4}$, Luiza Torres-Nunes ${ }^{2}$, \\ Vanessa da Silva Caiado ${ }^{(\mathbb{D}}$, Maria Eduarda de Souza Melo-Oliveira ${ }^{2,3}$, \\ Eliane de Oliveira Guedes de Aguiar ${ }^{2,4,5}$, Liszt Palmeira de Oliveira ${ }^{7}$, Alan de Paula Mozella ${ }^{7}$, \\ Laisa Liane Paineiras-Domingos ${ }^{2,4,5}$, Mario José dos Santos Pereira ${ }^{2}$, \\ Vinicius Layter Xavier ${ }^{8}{ }^{D}$, Ana Cristina Rodrigues Lacerda ${ }^{6}\left(\mathbb{D}\right.$, Vanessa Amaral Mendonça ${ }^{6}(\mathbb{D}$, \\ Borja Sañudo ${ }^{9}\left(\mathbb{D}\right.$, Adérito Seixas ${ }^{10}$, Redha Taiar ${ }^{11, *(\mathbb{D})}$, Danúbia da Cunha de Sá-Caputo ${ }^{2,4,5}$ \\ and Mario Bernardo-Filho ${ }^{2}$ iD
}

1 Programa de Pós-graduação em Fisiopatologia Clínica e Experimental, Faculdade de Ciências Médicas, Universidade do Estado do Rio de Janeiro, Rio de Janeiro 20550-170, Brazil; marciafernandesfisio@hotmail.com (M.C.M.-F.); eloamarconi@gmail.com (E.M.-M.); bessa.oliveira@gmail.com (B.B.M.d.O.); anacarol_coelho@hotmail.com (A.C.C.d.O.)

2 Laboratório de Vibrações Mecânicas e Práticas Integrativas-LAVIMPI, Departamento de Biofísica e Biometria, Instituto de Biologia Roberto Alcântara Gomes and Policlínica Piquet Carneiro, Universidade do Estado do Rio de Janeiro, Rio de Janeiro 20511-010, Brazil; meirelles.ale@gmail.com (A.G.d.M.); fisio.alinereis@hotmail.com (A.R.-S.); lumadaragu@gmail.com (L.F.F.d.S.); adrianaliriolavimpi@gmail.com (A.L.P.d.S.); drmarcogamafisioperito@gmail.com (M.A.d.S.G.); danielbatouli@gmail.com (D.B.-S.); silvarogy@hotmail.com (Y.T.d.S.); luiza-nunes@bol.com.br (L.T.-N.); vancaiado83@gmail.com (V.d.S.C.); mariaeduardaoliveira@hotmail.com (M.E.d.S.M.-O.); ellianeguedes@gmail.com (E.d.O.G.d.A.); laisanit@gmail.com (L.L.P.-D.); mario@anima.bio.br (M.J.d.S.P.); dradanubia@gmail.com (D.d.C.d.S.-C.); bernardofilhom@gmail.com (M.B.-F.)

3 Mestrado Profissional em Saúde, Medicina Laboratorial e Tecnologia Forense, Universidade do Estado do Rio de Janeiro, Rio de Janeiro 21941-901, Brazil

4 Programa de Pós-graduação em Ciências Médicas, Faculdade de Ciências Médicas, Universidade do Estado do Rio de Janeiro, Rio de Janeiro 20550-170, Brazil

5 Departamento de Fisioterapia, Faculdade Bezerra de Araújo, Rio de Janeiro 23052-180, Brazil

6 Centro Integrado de Pós-Graduação e Pesquisa em Saúde-CIPq/Saúde,

Faculdade de Ciências Biológicas e da Saúde, Universidade Federal dos Vales do Jequitinhonha e Mucuri-UFVJM, Diamantina 39100-000, Minas Gerais (MG), Brazil; lacerdaacr@gmail.com (A.C.R.L.); vaafisio@hotmail.com (V.A.M.)

7 Departamento de Especialidades Cirúrgicas, Faculdade de Ciências Médicas, Universidade do Rio de Janeiro, Rio de Janeiro 20550-170, Brazil; lisztpalmeira@yahoo.com.br (L.P.d.O.); apmozella@terra.com.br (A.d.P.M.)

8 Departamento de Estatística, Instituto de Matemática e Estatística, Universidade do Rio de Janeiro, Rio de Janeiro 20550-900, Brazil; viniciuslx@ime.uerj.br

9 Departamento de Educación Física y Deporte, Universidad de Sevilla, 41013 Sevilla, Spain; bsancor@us.es

10 Escola Superior de Saúde, Fundação Fernando Pessoa, 4249-004 Porto, Portugal; aderito@ufp.edu.pt

11 Université de Reims Champagne-Ardenne, 51100 Reims, France

* Correspondence: redha.taiar@univ-reims.fr 
Abstract: Introduction: Among chronic diseases, knee osteoarthritis (KOA) is a joint disease that causes important progressive alterations in the articular and periarticular structures, including synovial inflammation. Exercise has been suggested as an intervention to KOA individuals, and studies suggest that whole-body vibration (WBV) exercise decreases pain levels and favours the functionality of KOA individuals. Objective: The aim of the present study is to analyze the acute effects of WBV exercise on pain levels, functionality (Timed Up and Go (TUG test), anterior trunk flexion (ATF)), and rating of exertion of elderly obese KOA individuals. Methods: Thirty-seven individuals with KOA were allocated to a WBV exercise group (WBVEG), $n=19$ (15 females/4 males), and a control group (CG), $n=18$ (15 females/3 males). WBVEG performed one session of WBV exercise (11 min, using $5 \mathrm{~Hz}, 2.5,5.0$, and $7.5 \mathrm{~mm}, 0.12,0.25$, and $0.37 \mathrm{~g}$ ). Three bouts were performed (working time of $3 \mathrm{~min}$ and rest time of $1 \mathrm{~min}$ ) using a side-alternating vibrating platform (VP). The same position was used in CG; however, the VP was turned off and there was equipment coupled to the VP that emitted a sound like the vibrations. The pain level was evaluated using a visual analog scale (VAS). Functionality was evaluated with a TUG test and ATF. The rating of subjectively perceived exertion was measured with the category ratio CR-10 (BORG Scale CR-10), Results: A reduction of pain levels in WBVEG after the intervention $(p=0.001)$ and intergroups $(p=0.041)$ was found. A decrease of TUG test time in both groups $(p=0.001)$ and intergroups $(p=0.045)$ was found, while no statistical changes were observed in the Borg Scale score. Significant improvements of flexibility in both groups $(p=0.001)$ and intergroups $(p=0.043)$ were found. Conclusion: One session of WBV exercise can lead to important improvements in individuals with KOA, possibly triggered by physiological responses. However, more studies are needed, in this clinical context, to confirm these results.

Keywords: pain level; functionality; knee osteoarthritis; physical and rehabilitation medicine; whole-body vibration

\section{Introduction}

Knee osteoarthritis (KOA) may be a cause of chronic pain with progressive disability, causing a significant impact on the quality of life of individuals, mainly in the elderly [1-3]. KOA is considered as a chronic progressive joint disease that causes important changes in the articular and periarticular structures, including synovial inflammation [1-4].

To establish the diagnosis of KOA, several aspects must be considered, such as clinical history, physical examination, and analysis of radiographic evidence using the Ahlbäck criteria $[5,6]$. In symptomatic cases of $\mathrm{KOA}$, due to knee joint instability, affected individuals can manifest severe pain, decreased strength in the lower limbs, reduced balance, and slower gait, which can lead to an increased fall risk [7-12].

Women are more likely to develop KOA [7], and obesity is considered a risk factor for the worsening and development of KOA because of the mechanical stress in the joint $[1-4,7,8,13]$, and this can be a risk factor for the appearance of hip osteoarthritis (coxarthrosis) [13]. The high prevalence of KOA and the severe impact on the individual's functionality reflect the need for constant monitoring and development of strategies for obesity control, pain reduction, and improvement of functional symptoms in this population [1-4]. Numerous management strategies have been proposed for this disease, including pharmacological treatments, such as analgesics, nonsteroidal anti-inflammatory drugs, oral corticoids, glucosamine, chondroitin, and avocado, and nonpharmacological treatments, such as regular physical activity, aerobic exercise, weight loss, acupuncture, and physiotherapy. Intra-articular hyaluronic acid injection (viscosupplementation), intra-articular corticosteroid injection, and even surgical intervention have been recommended when necessary $[1-4,14]$.

The American College of Sports Medicine (ACSM) [15] and the American Heart Association (AHA) [16] suggest physical exercise as an important tool to maintain health by preventing and 
controlling chronic diseases, injuries, and other illnesses. Physical exercise and weight management have shown positive results for the improvement of KOA symptoms [17-20]. However, individuals with KOA have difficulty exercising in a regular way [18].

In this context, WBV exercises appear as a safe, accessible, and effective exercise for people with KOA. WBV exercise is generated when the individual is exposed to mechanical vibration produced by a vibrating platform (VP). Biomechanical parameters of WBV exercise (frequency (f), peak-to-peak displacement $(D)$, and peak acceleration (a peak)) need to be established and adjusted to the characteristics of the individuals [21]. Furthermore, the positioning of the individual on the VP, working time, session interval, and rest time must be clearly defined [21,22].

WBV exercise has been used in KOA patients to decrease pain levels and improve handgrip strength [23] and physical function [24]; however, the best protocol for these individuals has not been reported yet. It is also reported that WBV exercise improves trunk flexibility, gait speed, and handgrip strength in metabolic syndrome individuals $[25,26]$ and increases inflammatory markers in individuals with chronic obstructive pulmonary disease [27].

Considering the WBV exercise in the management of KOA, the aim of this present study is to analyze the effects of a single WBV exercise on the level of pain, functionality (Timed Up and Go (TUG test) and flexibility), and rating of perceived exertion in KOA individuals. It is hypothesized that acute WBV exercise would have beneficial effects on pain, functionality, and rating of perceived exertion in this population group.

\section{Materials and Methods}

\subsection{Trial Design}

This randomized study was performed to evaluate the acute effects of WBV exercise (WBVE) in KOA patients. The individuals were randomly allocated to a WBV exercise group (WBVEG) or a control group (CG). This work was approved by the Ethics Committee (CAAE n'198 26413.8.0000.5259), registered in the Brazilian Registry of Clinical Trials (ReBEC n ${ }^{\circ}$ RBR 738wng), and conducted according to the Declaration of Helsinki. The individuals were notified about all stages of this investigation, and they signed a specific informed consent form (ICF). The data analysis was done by a blinded researcher. Due to the great difficulty of blinding the individuals, this study was performed without blinding these individuals to the interventions.

\subsection{Inclusion Criteria}

Obese individuals (body mass index (BMI) $\geq 30 \mathrm{~kg} / \mathrm{m}^{2}$ ) [28], over 60 years old, diagnosed with unilateral or bilateral symptomatic primary KOA (individuals with self-reported complaints of pain and/or unilateral or bilateral stiffness of the knee joint), according to the American College of Rheumatology (ACR) [1] were included. The diagnosis was defined by the clinical staff of the Setor de Ortopedia e Traumatologia, Hospital Universitário Pedro Ernesto (HUPE), Rio de Janeiro, Brazil, following the Ahlbäck criteria (Ahlbäck classification, Grades 1, 2, 3, 4 and 5) [5,6].

\subsection{Exclusion Criteria}

Individuals with secondary osteoarthritis, asymptomatic KOA, with recent (six months) lower limb surgery, severe osteoporosis, severe hip arthrosis, hip or knee arthroplasty, unilateral or bilateral hip prosthesis, bilateral knee prosthesis, spine arthrodesis, periarticular metal implants and/or history of previous knee injury (meniscus, ligament, sprains), using corticosteroids for less than three months, viscosupplementation for at least six months, with cardiomyopathies and/or cardiac pacemaker, found in other rehabilitation programs, with clinical manifestations that do not allow exercise, with neurological and/or psychiatric disorders that generate "fear and/or panic" when performing WBV exercise, with three consecutive absences, or refusing to sign the consent form were excluded. 


\subsection{Randomization}

On the first day of this protocol, the randomization was performed using an electronic platform (http: //www.random.org) [29,30]. Forty numbers were generated: 20 to the control group (CG; green color) and 20 to whole-body vibration exercise group (WBVEG; blue color). The randomization was performed after the signing of the ICF. Depending on the color, the participant was allocated to a group in a blinded way.

\subsection{Participants}

Sixty-two individuals were recruited, but only 37 performed the protocol (18 in CG and 19 in WBVEG). The recruitment was performed at the Setor de Ortopedia e Traumatologia of HUPE from September 2019 to March 2020. Afterward, the individuals were taken to the Setor de Radiologia of HUPE to perform radiographic imaging. Participants were also advised not to use analgesics and nonsteroidal anti-inflammatory drugs (NSAIDs) during the interventions.

The selection process of the individuals involved radiographic exams that were performed on (i) knees (left and right) in an anteroposterior view, with load, bipedal support, and profile, (ii) axial patella, (iii) hip in the anteroposterior view, (iv) lumbosacral spine in anteroposterior view and orthostatic profile for the diagnosis of KOA. The exams were performed using a Shimadzu General Radiographic System X-ray Tube Stand FH-20HR (Shimadzu Corporation, Kyoto, Japan) and were reviewed by the clinical team of the Setor de Radiologia.

The Ahlbäck classification was used to evaluate the degree of radiographic affection and the development of KOA. Although this classification has some criticisms due to the possible disagreement among evaluators with different degrees of experience, it is more reproducible when carried out by a more experienced evaluator [31,32].

In this study, the interobserver variability for Ahlbäck's classification was performed through the analysis of radiographic images by three orthopedic physicians (HUPE) belonging to the Sociedade Brasileira de Ortopedia e Traumatologia. In case of disagreement, the physician with the highest academic degree and most years of experience decided on the variability of Ahlbäck's classification (Grade 1: joint space narrowing (less than $3 \mathrm{~mm}$ ); Grade 2: joint space obliteration; Grade 3: minor bone attrition $(0-5 \mathrm{~mm})$; Grade 4 : moderate bone attrition $(5-10 \mathrm{~mm})$; Grade 5: severe bone attrition (more than $10 \mathrm{~mm})$ ).

Where the evaluators considered the radiographic images to be of low technical quality, the examinations were repeated. After the diagnosis of KOA, the participants were referred to the Laboratório de Vibrações Mecânicas e Praticas Integrativas (LAVIMPI, UERJ) to begin the intervention protocol.

During all measurements and interventions, the supervisors were near the individual to clarify the procedures. The supervisors of WBVEG and CG were different physiotherapists.

\section{Measurements}

\subsection{The First Day of Evaluation}

In the first day, the following steps were performed: (i) signing of the ICF, (ii) randomization, (iii) anamnesis (age; sex; the practice of physical activity, where the individuals were asked whether they practiced physical activity or not; pharmacological treatment; nutritional monitoring, where the individuals were asked whether they performed nutritional monitoring or not; the existence of comorbidities), (iv) evaluation of the pain level using a visual analog scale (VAS), (v) evaluation of functional disability in the lumbosacral spine through the Oswestry Disability Index (ODI) questionnaire; (vi) evaluation of symptoms and functional disability of the lower limbs using the Lequesne's Functional Index (LFI) questionnaire. 
Pain Level Analysis

To assess pain, VAS [33] was used at three moments: (i) on the first day (during anamnesis), (ii) on the second day (before and after WBV intervention), and (iii) immediately after the participant got up from the chair without the help of the upper limbs. The individuals were asked to inform us on the knee pain level according to VAS (0-without pain; from 1 up to 3-mild; from 4 to 6-moderate; from 7 to 9-intense; 10-high pain; see Figure 1).

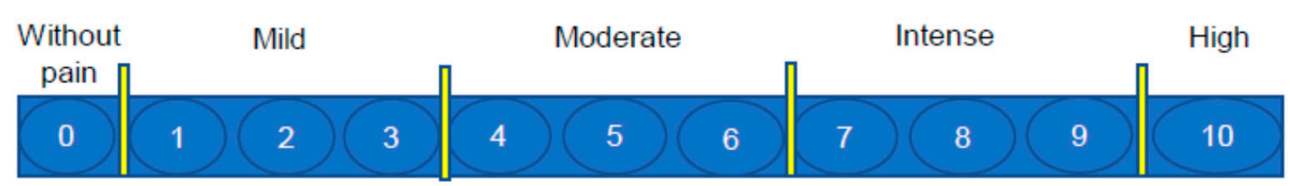

Figure 1. Visual analog scale used to evaluate pain level.

\subsection{Oswestry Disability Index}

For analysis of the functional disability in the lumbosacral spine, the ODI questionnaire [34] was used. This instrument has 10 questions, with 6 options to answer in each one. Higher scores are related to worse functionality.

\subsection{Lequesne's Functional Index}

For analysis of symptoms and functional disability of the lower limbs in individuals with KOA, the LFI questionnaire [35] was used. This analysis was performed on the first day. This instrument evaluates pain and discomfort related to the activities of daily living. This instrument has eleven questions about pain, discomfort, and function. Six questions are related to pain and discomfort (one for knee and another for hip), one is related to performance during walking, and four are related to hip or knee (according to the objective of evaluation) during daily activities. The scoring of the questions is done according to the difficulty of performing daily activities: ( 0 -without difficulty; 0.5-easily; 1.0 -with difficulty; 1.5 -very difficult; 2 -impossible). As to the total score, (i) from 1 to 4 means little commitment, (ii) from 5 to 7 means moderate commitment, (iii) from 8 to 10 means serious commitment, (iv) from 11 to 13 means very serious commitment, and (v) higher or equal than 14 means extremely serious commitment.

\subsubsection{The Second Day of Evaluation}

On the second day (before the intervention), the following steps were done: (i) measurement of height, (ii) measurement of body mass, (iii) measurement of waist circumference, (iv) determination of body mass index, (v) evaluation of gait with the Timed Up and Go test (TUG test), (vi) measurement of perceived effort (BORG scale), (vii) measurement of flexibility (anterior trunk flexion (ATF)), (viii) measurement of the pain level (VAS), and (ix) interventions (WBVEG or CG). After the intervention session (WBVEG or CG), the VAS, ATF, BORG scale, and TUG test assessments were performed again, in this sequence.

\subsubsection{Evaluation of Height and Waist Circumference}

For height measurements, a stadiometer (MIC 200 PPA, Micheletti, São Paulo, Brazil) was used, and WC was measured with an inelastic tape around the abdomen (umbilicus level) [36].

\subsubsection{Body Composition Analysis}

Body mass (BM) and body mass index (BMI) were obtained with an InBody 370 System Composition Analyzer, Model BPM040S12FXX (InBody Co., Ltd., Seoul, Korea). To perform these measurements, participants wore light clothing (preferably with a bathing suit), without objects such as watches, rings, earrings. The use of diuretics was discontinued $24 \mathrm{~h}$ prior to the examination, and the 
consumption of drinks and food was suspended for $8 \mathrm{~h}$ before the exam. The practice of physical activity was suspended $12 \mathrm{~h}$ before the assessment [37-39].

\subsubsection{TUG Test}

To assess functionality, the TUG test [40] was performed. All participants got up from a chair, with arms crossed over the chest area, walked $3 \mathrm{~m}$, turned, walked back to the chair, and sat down. Two attempts were performed, and the time of the TUG test was recorded in a proper form, timed in seconds (s) with a digital timer (Cronobio, Modelo SW2018, São Paulo, SP, Brazil). This test was performed before and after the intervention. The time to perform the test was registered, and higher performance times were related to worse performance.

\subsection{Flexibility}

The flexibility analysis was performed using the ATF test [26]. All the participants were positioned in the orthostatic position, with feet together. They were asked to flex their trunk (as much as possible) without knee flexion and/or extension of the neck. During the trunk flexion, the distance $(\mathrm{cm})$ between the middle finger to the floor was obtained using a ruler by the supervisor. Higher distance values between the middle finger and the floor were related to worse performance in the test. The ATF test was performed before and after the intervention.

\subsection{Subjective Perceived Exertion Rating}

To assess the rating of perceived exertion, the category ratio (Borg Scale CR-10) was used [41]. The individuals were asked to refer to their effort perception, considering the score where 0 is related to "without effort" and 10 is related to "maximum effort-exhaustion" (Figure 2). The Borg Scale CR-10 was used before and after the interventions, and it was used to estimate the subjects' effort perception to perform the intervention.

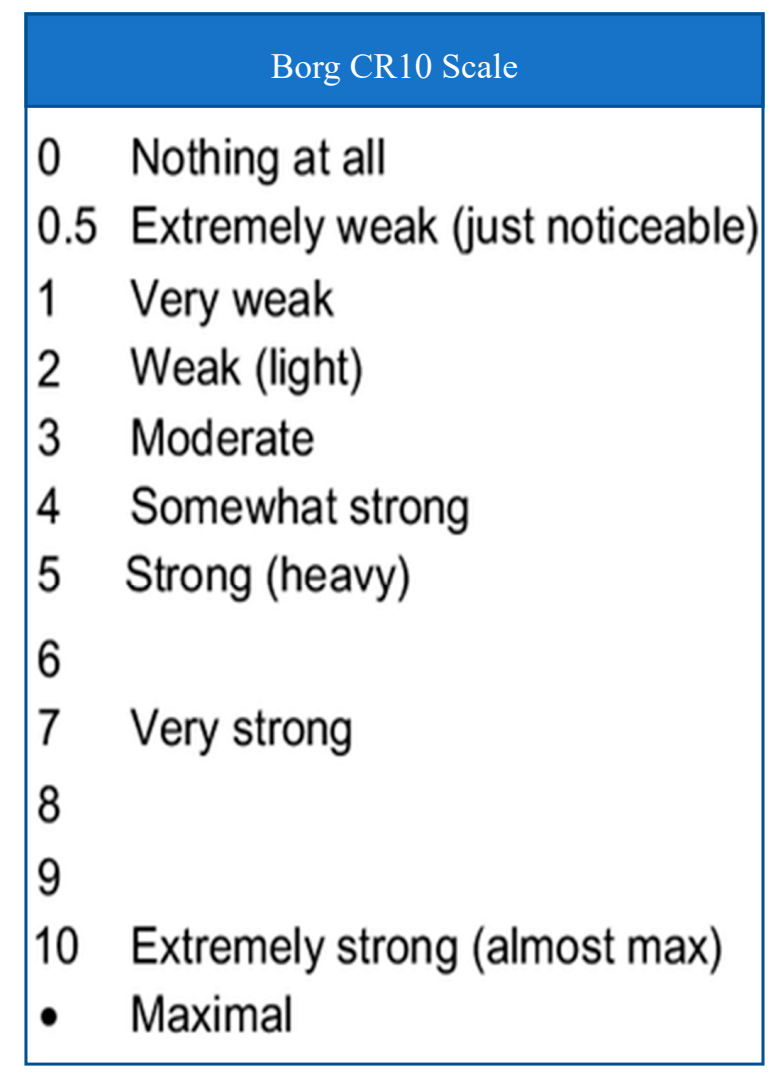

Figure 2. Score of the Borg CR-10 scale used to evaluate the rating of perceived exertion. 


\subsection{Protocol Interventions}

The individuals of WBVEG and CG did not know the protocol of the interventions, which were carried out in separate rooms, and they had no contact with each other. Therefore, the individuals of WBVEG did not know that CG used a custom platform that was turned off (with an equipment fixes in the VP that emitted a sound when the VP was turned on). In the same way, the individuals of CG were not informed that WBVEG used a custom platform that was turned on (with the equipment plugged in and without a sound like a platform that is turned on).

\subsection{WBV Exercise Protocol}

Participants were instructed by the supervisor to sit in a chair in front of the VP in a comfortable position, with bare feet resting on the VP, arms extended, and hands on the knees, with an average flexion angle between $100^{\circ}-120^{\circ}$ (Goniometer Carci, Instituto São Paulo, São Paulo, Brazil).

The VP was a side-alternating (Nova Plate Fitness Evolution, DAF Produtos Hospitalares Ltda., SP, Brazil) device. The participants performed one session of WBV exercise (11 min of total time), using $5 \mathrm{~Hz}, 2.5,5.0$, and $7.5 \mathrm{~mm}$, and $0.12,0.25$, and $0.37 \mathrm{~g}$, considering frequency, $D$, and acceleration peak, respectively. The exercise was performed in 3 bouts $(3 \mathrm{~min}$ of working time and $1 \mathrm{~min}$ of rest time; Figure 3) [42]. A dark-colored plate was attached to the VP display so that the participant did not identify the time and frequency used in the intervention [21,22].

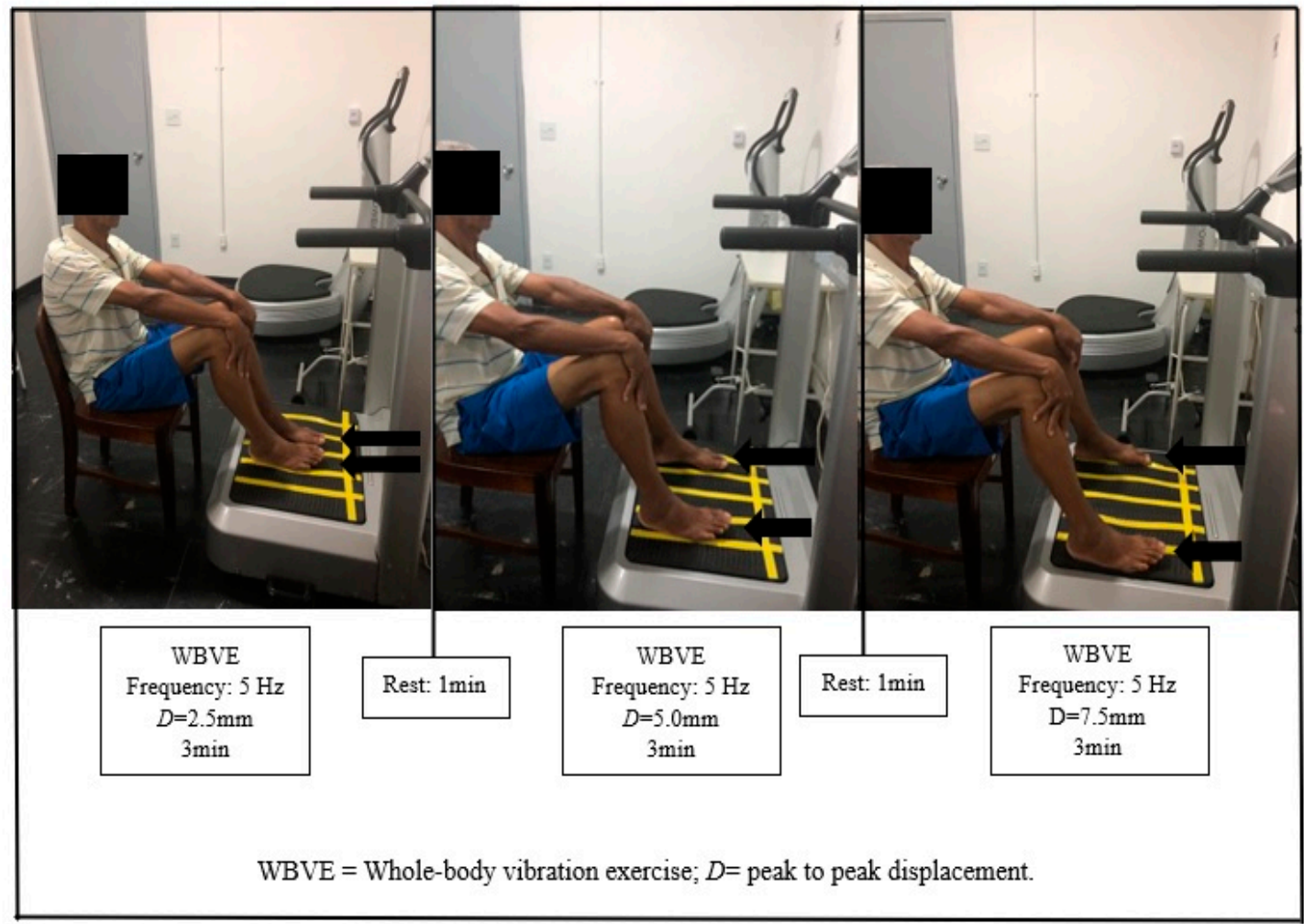

Figure 3. Whole-body vibration exercise intervention.

The frequency of the mechanical vibration was measured by an accelerometer (EMG832WF Digital Accelerometer, EMG System do Brazil, São José dos Campos, SP, Brazil).

The supervisor monitored all procedures and advised the participant to report any discomfort (nausea, dizziness) that occurred during the execution of the intervention. The supervisor performed the data collection of heart rate, respiratory rate, and blood pressure before and after the intervention for the participant's safety [25]. 


\subsection{CG Protocol}

Participants were submitted to the same protocol as WBVEG, but with the VP turned off. During the intervention, equipment was coupled with the VP to emit a sound like a mechanical vibration. This equipment was positioned behind the VP (Figure 4). The frequency of the VP was $0 \mathrm{~Hz}$.
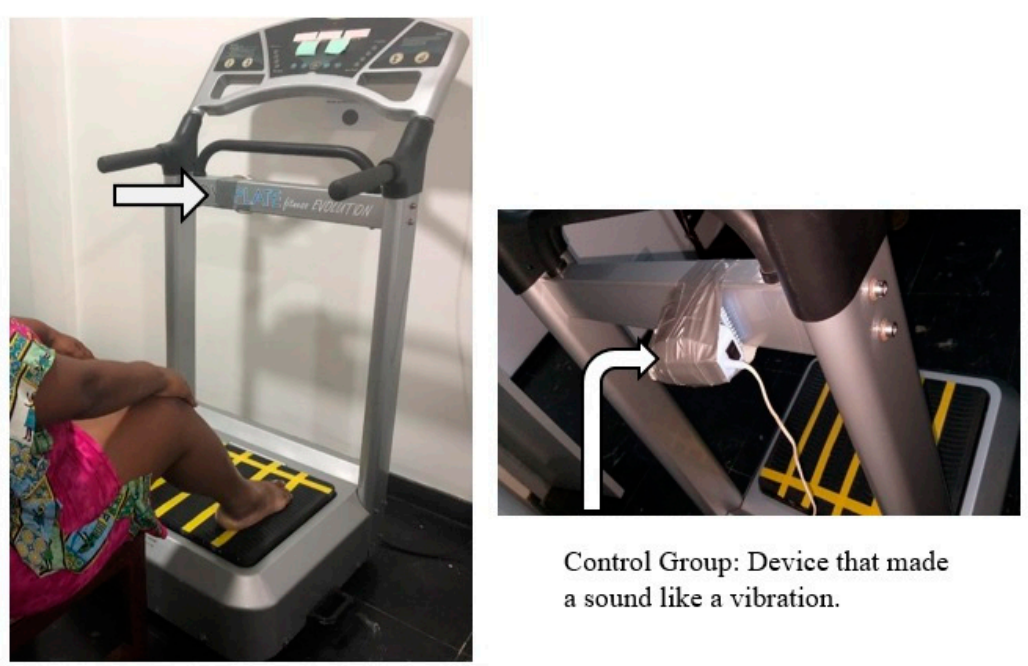

Control Group: Device that made a sound like a vibration.

Figure 4. Control group intervention.

The steps of the study protocol are presented in Figure 5.

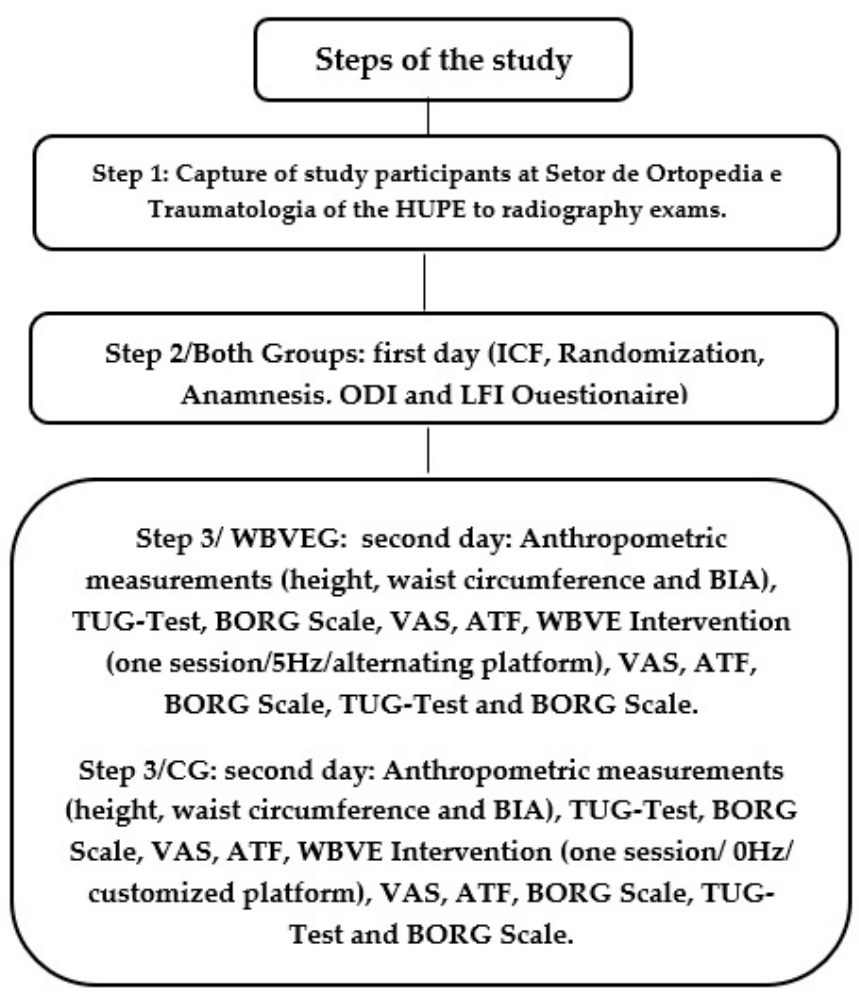

HUPE- Hospital Universitário Pedro Ernesto; ICF- Informed Consent Form. ODI- Oswestry Disability Index; LFI - Lequesne's Functional Index; WBVE- Whole-body vibration exercises; BIA- Bioelectrical Impedance Analysis; TUG-Test- Timed up and go test; VAS- Visual Analogue Scale; ATF- Anterior trunk flexion; CG- Control Group.

Figure 5. Steps performed in the study protocol. 


\subsection{Sample Size}

Sample size was determined using the formula by Miot [43] to an infinite population. The variable TUG test was used due to its importance in the functionality context of this study. Considering a standard deviation of 0.77 and a mean of 6.58 [24], sixteen individuals in each group were needed.

\subsection{Data Analysis}

Statistical analysis was conducted using the GraphPad Prism 6.0 program. Nonparametric tests to compare the characteristics of participants of the groups were applied. A paired Wilcoxon signed-rank test to compare the difference before and after interventions was applied. For analysis between the groups (CG and WBVEG), the difference $(\Delta)$ between before and after interventions was calculated, and the Mann-Whitney test was used to analyze intergroup differences (CG vs WBVEG). For all data, the mean and the standard error were reported. Statistical significance was set at $p \leq 0.05$.

\section{Results}

Sixty-two individuals were recruited, and 37 (18 in CG and 19 in WBVEG) fulfilled the eligibility criteria. Sixteen volunteers did not meet the inclusion criteria (14 participants did not have KOA and 2 had rheumatoid arthritis), and 9 were excluded (3 participants used corticosteroids, 1 with a tumor on the chest, 1 with a hip prosthesis, 1 with osteonecrosis, 1 with Behcet's disease, 1 with recent surgery on the lumbar spine, and 1 with recent surgery on the anterior cruciate ligament). All participants of both groups completed the study, and no discomfort was reported. The development of the study, considering each stage, is shown in Figure 6.

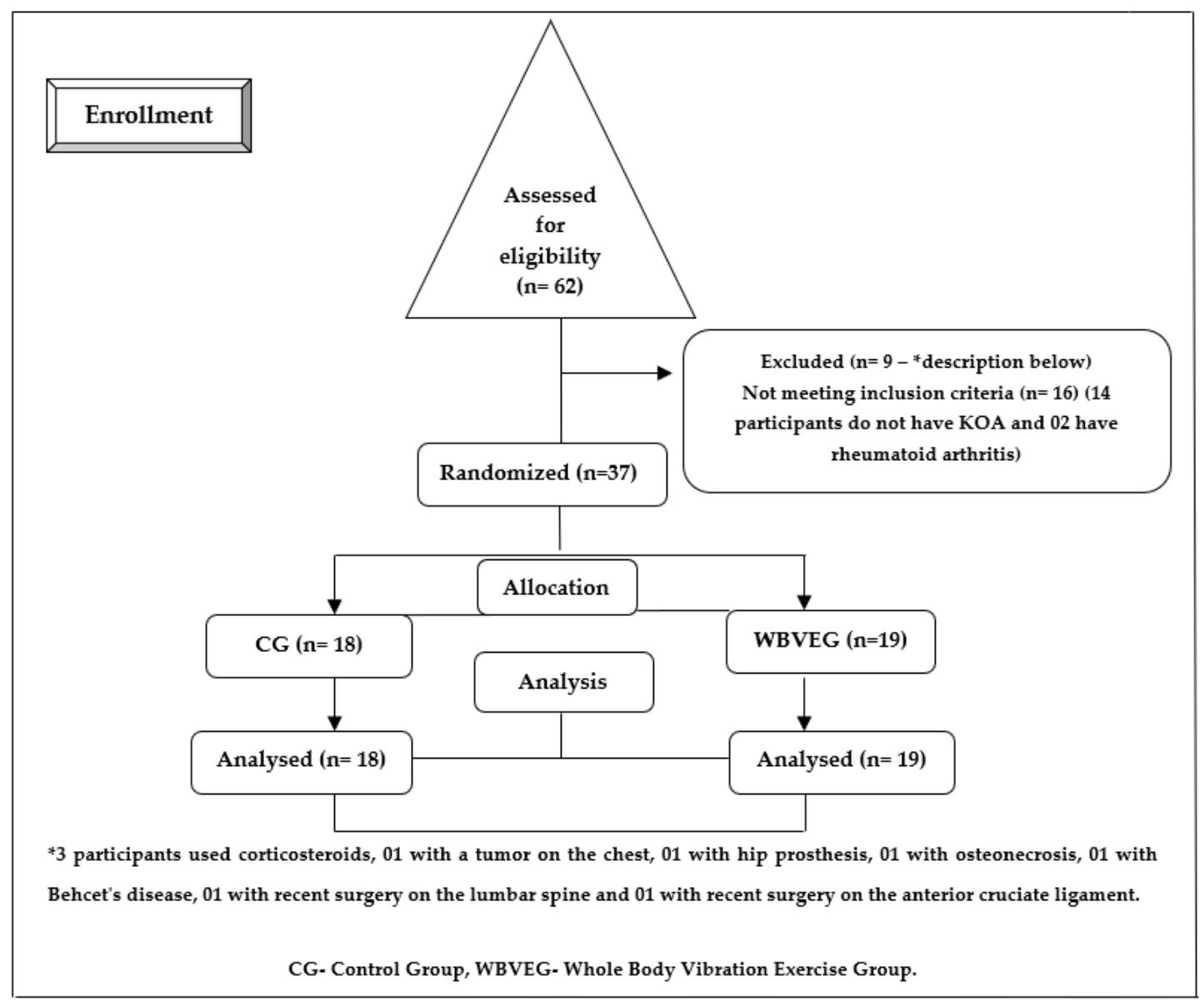

Figure 6. Flow diagram with the development of the study. 
Considering WBVEG, 2 individuals did not have bilateral KOA, 10 individuals presented Grade 1, 5 individuals presented Grade 2, and 3 individuals presented Grade 5 in the right knee. In the left knee, 10 individuals were Grade 1, 5 individuals were Grade 2, 1 individual was Grade 3, and 2 individuals were Grade 5.

Concerning CG, all individuals had bilateral KOA, 13 individuals presented Grade 1, 2 individuals presented Grade 2, 1 individual presented Grade 3, and 2 individuals presented Grade 4 in the right knee. In the left knee, 11 individuals were Grade 1, 4 individuals were Grade 2, 1 individual was Grade 3, 1 individual was Grade 4, and 1 individual was Grade 5.

Analyzing the baseline characteristics of KOA subjects regarding sex, waist circumference, body mass, body mass index, height, age, ODI, LFI, and VAS, no significant differences were observed between CG and WBVEG, confirming the homogeneity of the sample (Table 1).

Table 1. Baseline characteristics of the knee osteoarthritis individuals of WBVEG and CG.

\begin{tabular}{cccc}
\hline & CG $(\boldsymbol{n}=\mathbf{1 8})$ & WBVEG $(\boldsymbol{n = 1 9 )}$ & \\
\cline { 2 - 3 } & Mean (SE) & Mean $(\mathrm{SE})$ & \\
\hline Sex & $\mathrm{F}=15 / \mathrm{M}=3$ & $\mathrm{~F}=15 / \mathrm{M}=4$ & 1.000 \\
Age (year) & $68.06(2.02)$ & $62.32(2.52)$ & 0.112 \\
Height (m) & $1.54(0.01)$ & $1.58(0.01)$ & 0.123 \\
Body mass (kg) & $75.67(3.18)$ & $86.15(4.50)$ & 0.091 \\
Waist circumference (cm) & $103.9(2.54)$ & $106.9(3.65)$ & 0.851 \\
BMI (kg/m $\left.{ }^{2}\right)$ & $31.71(1.22)$ & $34.75(2.10)$ & 0.420 \\
ODI (\%) & $30.61(2.86)$ & $35.39(2.95)$ & 0.201 \\
LFI (score) & $11.81(3.66)$ & $11.85(3.89)$ & 0.989 \\
VAS (0-10) & $4.33(0.52)$ & $4.36(0.60)$ & 0.981 \\
\hline
\end{tabular}

F-female; M-male; BMI-body mass index; VAS—visual analog scale; ODI-Oswestry Disability Index; LFI-Lequesne's Functional Index; WBVEG—whole-body vibration exercise group; CG—control group; SE-standard error; $p \leq 0.05$.

Considering the radiographic images, no statistical difference was found between CG and WBVEG as well: (i) Ahlbäck classification—knees right $(p=0.105)$ and left $(p=0.840)$, (ii) coxarthrosis—hips right $(p=0.696)$ and left $(p=1.000)$, and (iii) Lumbosacral spine-decrease in intersomatic space $(p=0.696)$, interapophyseal arthrosis $(p=1.000)$, degenerative spondylolisthesis $(p=1.000)$, and degenerative scoliosis $(p=1.000)$.

Analyzing nutritional monitoring $(p=1.000)$ and practice of physical activity $(p=0.453)$, similarly, no difference was found between CG and WBVEG.

Concerning existing comorbidities, again, no difference was found between CG and WBVEG: arterial hypertension $(p=0.277)$ and type 2 diabetes mellitus $(p=0.180)$.

Considering the pain level assessment (VAS), there was a significant decrease after the WBVEG intervention and between groups (Table 2).

Table 2. Pain level, functionality (TUG test and anterior trunk flexion (ATF)), and subjectively perceived exertion rating (BORG Scale CR-10) assessments before and after the intervention and between groups.

\begin{tabular}{cccccccc}
\hline & $\begin{array}{c}\text { CG before } \\
\text { Mean (SE) }\end{array}$ & $\begin{array}{c}\text { CG after } \\
\text { Mean (SE) }\end{array}$ & $p$-Value & $\begin{array}{c}\text { WBVEG before } \\
\text { Mean (SE) }\end{array}$ & $\begin{array}{c}\text { WBVEG after } \\
\text { Mean (SE) }\end{array}$ & $p$-Value & $p$-Value \# \\
\hline VAS (0-10) & $4.11(0.56)$ & $3.94(0.57)$ & 0.564 & $4.58(0.62)$ & $3.23(0.61)$ & $0.001 *$ \\
TUG-test (s) & $14.87(0.88)$ & $13.84(0.78)$ & $0.002 *$ & $19.42(1.96)$ & $16.44(1.50)$ & $<0.041 *$ \\
BORG SCALE (score) & $1.66(0.56)$ & $1.58(0.51)$ & $>0.999$ & $2.15(0.54)$ & $2.15(0.54)$ & 0.627 & $0.045 *$ \\
ATF (cm) & $17.53(2.54)$ & $16.31(2.53)$ & 0.103 & $18.81(2.08)$ & $14.64(2.05)$ & $<0.001 *$ & $0.043 *$ \\
\hline
\end{tabular}

TUG test-Time Up and Go test; BORG Scale-subjectively perceived exertion rating; WBVEG-whole-body

vibration exercise group; CG—control group; SE—-standard error; ${ }^{*} p$-value $\leq 0.05$; \# intergroups.

Regarding the functionality assessment (TUG test and ATF measurement), significant improvements were found before and after the CG and WBVEG interventions and between groups in the TUG test, and a significative difference was obtained before and after the WBVEG intervention and between groups in the ATF test (Table 2). 
The data on the rating of perceived exertion (BORG scale) did not present significative differences in any comparison (Table 2).

\section{Discussion}

The aim of the study is to analyze the acute effects of WBVE on the level of pain, functionality, and rating of perceived exertion in KOA individuals. The results suggest that one session of WBW exercises, with low frequency $(5 \mathrm{~Hz})$ and peak to peak displacement $(2.5,5.0$, and $7.5 \mathrm{~mm})$, was able to reduce the pain level and time taken to perform the TUG test and improve flexibility (functionality) without altering the subjective perception of the effort of these individuals. These results corroborate the findings of Neto et al. [42], who showed that one session of WBV exercises with the same protocol, alone or in combination with auriculotherapy, decreased the pain of individuals with KOA.

All participants that completed the protocol did not report adverse effects. During the protocol, participants did not use analgesics and NSAIDs. Moreover, it is worth mentioning that this study simultaneously analyzed the knee (left and right) in three views (anteroposterior projection with load, bipedal support and profile, and patella axial), the hip (anteroposterior projection), and the lumbosacral spine (anteroposterior projection and orthostatic profile)), which makes it relevant for the diagnosis and study of KOA. No significant differences were found in CG and WBVEG participants in the radiographic images.

Considering nutritional monitoring, the practice of physical activity, and existing comorbidities, no differences were found between groups.

Regarding the functional capacity of KOA individuals, the time required to perform the TUG test was improved in CG and WBVEG, but the performance of WBVE was better than CG and the performance in the ATF test was improved only in WBVEG. A study reported that the transmission of mechanical vibration to the body (during WBVE) stimulates the primary endings of the muscle spindles, activating $\alpha$-motor neurons, resulting in muscle contractions (tonic vibration reflex) [44]. Thus, the lower limbs are stimulated, and the improvement in strength, proprioception, and execution of functional movements $[45,46]$, as observed through the TUG test, could be possible. Moreover, changes in the viscoelastic properties after vibration could explain the improvement in the ATF test in WBVEG.

Hsião et al. [47], using WBVE (vertical platform) for 2 to 3 days (from 8 to $10 \mathrm{~Hz}, 2 \mathrm{~mm}$ ) reported no significant difference in functional performance (using the TUG test) in the range of motion of the knee joint and in the reduction of pain in the postoperative period after total knee arthroplasty. Mikami et al. [48] described that WBVE improved knee muscle strength, dynamic balance, and TUG-test performance in healthy adult females. This protocol was performed 3 times a week for 12 sessions, with $10 \mathrm{~Hz}$ and $4.5 \mathrm{~mm}$, using a rotating-type WBV device.

The level of pain was reduced only in WBVEG. The mechanisms involved in the reduction of pain can be explained according to vibration-induced sensory inhibition related to peripheral (a sensory inhibition would be linked to the gate control theory of pain) and central levels (justified by the stimulation of areas responsible for processing pain and vibrotactile sensations in the somatosensory cortex of the brain) [36,49].

Pain is a cause of disability in individuals with KOA [1-3], and physical exercise is a very promising strategy to improve the symptoms of individuals affected by this disease [17-20]. Studies suggest that WBV exercise may be an effective and safe form of exercise for this population $[23,24,43]$. However, scientific evidence is still scarce and inconclusive in this population due to the diversity of WBV exercise protocols.

Regarding the pain level, the findings described in the current work are in line with Zafar et al. [50], who reported a decrease in pain and an improvement in the functionality of individuals with KOA after WBV. However, Wang et al. [51] observed that WBV exercise improved function but not pain and joint stiffness in individuals with KOA. Li et al. [52] did not present significant differences in the pain level and physical function of individuals with KOA after an 8-week protocol with a vertical VP 
(squat training position, $20 \mathrm{~Hz}$, and $2 \mathrm{~mm}$ ). In addition, Ferreira et al. [53] reported in an umbrella review that WBV exercises achieved moderate evidence for the improvement of KOA symptoms, but as a complementary intervention. Sá-Caputo et al. [25] and Paiva et al. [26], in metabolic syndrome individuals, also observed that a session of WBV exercises (frequency $5 \mathrm{~Hz}$, with the same peak to peak displacement and position) decreased the level of pain, ATF, and the subjective perception of effort. Simão et al. [54], in a different protocol, observed that vibratory training plus squat training (12 weeks, vertical platform, frequency from 35 to $40 \mathrm{~Hz}, 4 \mathrm{~mm}$, and from 2.78 to $3.26 \mathrm{~g}$ ) improved balance (static and dynamic), gait, and self-perception of pain in the elderly with KOA. However, Salmon et al. [55] observed that a WBV session on a triplanar (vertical) VP, with $35 \mathrm{~Hz}$ and the same peak-to-peak displacements, in a standing position with the knees slightly bent, did not improve the TUG test and did not affect the knee pain levels of individuals with KOA; two participants did not finish the protocol due to knee pain.

To our knowledge, this is the first work that has investigated the effects of WBVE on pain levels, functionality, and perception of effort. Thus, the pain reduction and improvement in functionality, without changing the perception of the effort of individuals with KOA, need to be confirmed in well-designed longitudinal randomized controlled trials. Considering the evaluation of acute effects, in this current study, the tests before and after WBVEs were performed in a 10-min time window. It would also be interesting to analyze, in future studies, how long the acute effects are maintained, which was not possible in the present study.

The strengths of the current work are the outcomes (pain level, functionality (TUG test), and flexibility), which are meaningful for the studied population, the rating of perceived exertion of KOA individuals, and the protocol with low frequency $(5 \mathrm{~Hz}$; with the individual in a sitting position in an ancillary chair to reduce the impact of the mechanical vibration overloading the knee joint). This position, in addition to offering the participant the possibility of comfortable and safe exercise, promotes better adherence to the proposed protocol since pain limits these individuals from the practice of regular physical activity. On the other hand, individuals with KOA have a higher risk of falls [10], which may compromise the participant's safety during the protocol in other positions. The results of this investigation are promising and can be considered of clinical importance in the treatment of KOA individuals.

The limitations of this study are related to (i) the use of only one frequency in the intervention, (ii) the sole use of Ahlbäck's classification to evaluate the degree of KOA, (iii) the use of the LFI questionnaire considering both knees (not considering right and left knees separately), (iv) not using nuclear magnetic resonance imaging to evaluate the bone structure, $(\mathrm{v})$ the vibration given to each patient, which was at the same level for every age, body mass, and intervention time (these conditions could be considered as bias), and (vi) the duration of the results, which was not investigated in a follow-up session. However, important findings were obtained.

\section{Conclusions}

One WBV exercise session, with a frequency of $5 \mathrm{~Hz}$, peak-to-peak displacement of 2.5, 5.0, and 7.5 $\mathrm{mm}$, and peak acceleration of $0.12,0.25$, and $0.37 \mathrm{~g}$, was used in the protocol. The exercise was performed for three bouts ( 3 min working time, and 1 min rest time) in a side-alternating VP. This protocol was able to reduce the pain level and improve functionality without changing the perception of the effort of individuals with KOA. In this work, acute effects were evaluated, and the duration of the responses (minute, hour, day) is not presented. Thus, further studies, such as randomized clinical trials, are needed to confirm the persistence of these effects in the long term.

Author Contributions: M.C.M.-F., E.M.-M., D.d.C.d.S.-C., and M.B.-F. participated in the design of the study, as well as prepared the manuscript. A.L.P.d.S., Y.T.d.S., A.G.d.M., L.F.F.d.S., M.E.d.S.M.-O., V.d.S.C., L.T.-N., L.P.d.O., and A.d.P.M. selected participants according to the exclusion criteria, as well as recorded assessments, interventions performed, and data on their own forms. D.B.-S., M.A.d.S.G., and B.B.M.d.O. organized all data in Excel, performed the statistical analysis, and elaborated on the tables. A.C.C.d.O., E.d.O.G.d.A., L.L.P.-D., M.J.d.S.P., V.L.X., and A.R.-S. wrote the abstract, corrected the English grammar, and suggested some changes in 
the text on the study, statistical analysis, tables, and some references. A.C.R.L., V.A.M., B.S., A.S., R.T., D.d.C.d.S.-C., and M.B.-F. created the final version of the manuscript. All authors have read and agreed to the published version of the manuscript.

Funding: This work was supported by Conselho Nacional de Desenvolvimento Científico e Tecnológico (CNPq) and Fundação de Amparo à Pesquisa do Estado do Rio de Janeiro (FAPERJ).

Conflicts of Interest: The authors declare no conflict of interest (financial, political, ideological, personal, academic, intellectual, commercial, or religious) in relation to this manuscript.

\section{References}

1. Kolasinski, S.L.; Neogi, T.; Hochberg, M.C.; Oatis, C.; Guyatt, G.; Block, J.; Callahan, L.; Copenhaver, C.; Dodge, C.; Felson, D.; et al. 2019 American College of Rheumatology/Arthritis Foundation Guideline for the Management of Osteoarthritis of the Hand, Hip, and Knee. Arthritis Rheumatol. 2020, 72, 220-233. [CrossRef] [PubMed]

2. Bannuru, R.R.; Osani, M.; Vaysbrot, E.; Arden, N.; Bennell, K.; Bierma-Zeinstra, S.; Kraus, V.; Lohmander, L.; Abbott, J.; Bhandari, M.; et al. OARSI guidelines for the non-surgical management of knee, hip, and polyarticular osteoarthritis. Osteoarthr. Cartil. 2019, 27, 1578-1589. [CrossRef]

3. De Campos, G.C.; De Sousa, E.B.; Hamdan, P.C.; Júnior, C.S.D.A.; Tieppo, A.M.; De Rezende, M.U.; Alchaar, A.A.D.A.; Pinheiro, C.B.; Rocha, E.D.M.C.; Cunha, F.G.; et al. Brazilian consensus statement on viscosupplementation of the knee (COBRAVI). Acta Ortopédica Brasileira 2019, 27, 230-236. [CrossRef] [PubMed]

4. De Rezende, M.U.; De Campos, G.C. Is osteoarthritis a mechanical or inflammatory disease? Rev. Bras. Ortop. 2013, 48, 471-474. [CrossRef]

5. Ahlbäck, S. Osteoarthrosis of the knee. A radiographic investigation. Acta Radiol. Diagn. 1968, 277, 7-72.

6. Keyes, G.W.; Carr, A.J.; Miller, R.K.; Goodfellow, J.W. The radiographic classification of medial gonarthrosis. Correlation with operation methods in 200 knees. Acta Orthop Scand 1992, 63, 497-501. [CrossRef]

7. McAlindon, T.; Bannuru, R.R.; Sullivan, M.; Arden, N.K.; Berenbaum, F.; Bierma-Zeinstra, S.; Hawker, G.A.; Henrotin, Y.; Hunter, D.J.; Kawaguchi, H.; et al. OARSI guidelines for the non-surgical management of knee osteoarthritis. Osteoarthr. Cartil. 2014, 22, 363-388. [CrossRef]

8. Altman, R.D.; Fries, J.F.; Bloch, D.A.; Carstens, J.; Mb, T.C.D.; Genant, H.; Gofton, P.; Groth, H.; McShane, D.J.; Murphy, W.A.; et al. Radiographic assessment of progression in osteoarthritis. Arthritis Rheum. 1987, 30, 1214-1225. [CrossRef]

9. Apold, H.; Meyer, H.E.; Nordsletten, L.; Furnes, O.; Baste, V.; Flugsrud, G. Risk factors for knee replacement due to primary osteoarthritis, a population based, prospective cohort study of 315,495 individuals. BMC Musculoskelet. Disord. 2014, 15, 217. [CrossRef]

10. Soh, S.-E.; Barker, A.L.; Morello, R.; Ackerman, I.N. Applying the International Classification of Functioning, Disability and Health framework to determine the predictors of falls and fractures in people with osteoarthritis or at high risk of developing osteoarthritis: Data from the Osteoarthritis Initiative. BMC Musculoskelet. Disord. 2020, 21, 138. [CrossRef]

11. Foley, S.; Lord, S.R.; Srikanth, V.; Cooley, H.; Jones, G. Falls risk is associated with pain and dysfunction but not radiographic osteoarthritis in older adults: Tasmanian Older Adult Cohort study. Osteoarthr. Cartil. 2006, 14, 533-539. [CrossRef] [PubMed]

12. Fisher, N.M.; Pendergast, D.R. Reduced muscle function in patients with osteoarthritis. Scand. J. Rehabil. Med. 1997, 29, 213. [PubMed]

13. Silva, N.A.; Montadon, A.C.O.S.; Cabral, M.V.S.P. Peripheral degenerative joint diseases. Einsten. São Paulo. 2008, 66 (Suppl. 1), S21-S28.

14. Rezende, M.U.; de Campos, G.C. Viscosuplplementation. Rev. Bras. Ortop. 2012, 47, 160-164. [CrossRef] [PubMed]

15. Donnelly, J.E.; Blair, S.N.; Jakicic, J.M.; Manore, M.M.; Rankin, J.W.; Smith, B.K. Appropriate Physical Activity Intervention Strategies for Weight Loss and Prevention of Weight Regain for Adults. Med. Sci. Sports Exerc. 2009, 41, 459-471. [CrossRef]

16. American Heart Association. Available online: http:https://www.heart.org/en/healthy-living/fitness/fitnessbasics/aha-recs-for-physical-activity-in-adults (accessed on 17 April 2020). 
17. Gay, C.; Chabaud, A.; Guilley, E.; Coudeyre, E. Educating patients about the benefits of physical activity and exercise for their hip and knee osteoarthritis. Systematic literature review. Ann. Phys. Rehabil. Med. 2016, 59, 174-183. [CrossRef]

18. De Rooij, M.; Van Der Leeden, M.; Cheung, J.; Van Der Esch, M.; Häkkinen, A.; Haverkamp, D.; Roorda, L.D.; Twisk, J.; Vollebregt, J.; Lems, W.F.; et al. Efficacy of Tailored Exercise Therapy on Physical Functioning in Patients with Knee Osteoarthritis and Comorbidity: A Randomized Controlled Trial. Arthritis Rheum. 2017, 69, 807-816. [CrossRef]

19. Tittlemier, B.J.; Wittmeier, K.D.; Webber, S.C. Quality and content analysis of clinical practice guidelines which include nonpharmacological interventions for knee osteoarthritis. J. Eval. Clin. Pract. 2020. [CrossRef]

20. Dell'Isola, A.; Jönsson, T.; Ranstam, J.; Dahlberg, L.E.; Hansson, E.E. Education, Home Exercise, and Supervised Exercise for People with Hip and Knee Osteoarthritis As Part of a Nationwide Implementation Program: Data from the Better Management of Patients With Osteoarthritis Registry. Arthritis Rheum. 2020, 72, 201-207. [CrossRef]

21. Rauch, F.; Sievanen, H.; Boonen, S.; Cardinale, M.; Degens, H.; Felsenberg, D.; Roth, J.; Schoenau, E.; Verschueren, S.; Rittweger, J. Reporting whole-body vibration intervention studies: Recommendations of the International Society of Musculoskeletal and Neuronal Interactions. J. Musculoskelet. Neuronal Interact. 2010, 10, 193-198.

22. Rittwger, J. Vibration as an exercise modality: How it may work, and what its potential might be. Eur. J. Appl. Physiol. 2010, 108, 877-904. [CrossRef] [PubMed]

23. Moreira-Marconi, E.; Dionello, C.F.; Morel, D.S.; Sá-caputo, D.C.; Souza-Gonçalves, C.R.; Paineira-Domingos, L.L.; Silva-Teixeira, Y.; dos Santos Pereira, M.J.; Bernardo-Filho, M. Whole body vibration and auriculotherapy improve handgrip strength in individuals with knee osteoarthritis. J. Tradit. Chin. Med. 2019, 39, 707-715.

24. Lai, Z.; Lee, S.; Hu, X.; Wang, L. Effect of adding whole-body vibration training to squat training on physical function and muscle strength in individuals with knee osteoarthritis. J. Musculoskelet. Neuronal Interact. 2019, 19, 333-341. [PubMed]

25. Sá-Caputo, D.; Paineiras-Domingos, L.L.; Oliveira, R.; Neves, M.F.; Brandão, A.; Marin, P.J.; Sañudo, B.; Furness, T.; Taiar, R.; Bernardo-Filho, M. Acute Effects of Whole-Body Vibration on the Pain Level, Flexibility, and Cardiovascular Responses in Individuals With Metabolic Syndrome. Dose-Response 2018, 16. [CrossRef] [PubMed]

26. Paiva, P.C.; Figueiredo, C.A.; Reis-Silva, A.; Francisca-Santos, A.; Paineiras-Domingos, L.L.; Martins-Anjos, E.; Melo-Oliveira, M.E.S.; Lourenço-Revelles, G.M.G.; Moreira-Marconi, E.; Guedes-Aguiar, E.O.; et al. Acute and Cumulative Effects with Whole-Body Vibration Exercises Using 2 Biomechanical Conditions on the Flexibility and Rating of Perceived Exertion in Individuals With Metabolic Syndrome: A Randomized Clinical Trial Pilot Study. Dose-Response 2019, 17. [CrossRef]

27. Lage, V.; Lacerda, A.C.R.; Neves, C.D.C.; Chaves, M.G.A.; Soares, A.A.; Lima, L.P.; Martins, J.B.; Matos, M.A.; Vieira, É.L.M.; Teixeira, A.L.; et al. Acute Effects of Whole-Body Vibration on Inflammatory Markers in People with Chronic Obstructive Pulmonary Disease: A Pilot Study. Rehabil. Res. Pract. 2018, 2018, 1-7. [CrossRef]

28. World Health Organization. Physical Status: The Use and Interpretation of Anthropometry; WHO Technical Report Series, 854; World Health Organization: Geneva, Swizterland, 1995.

29. Schulz, K.F.; Altman, D.G.; Moher, D. CONSORT 2010 Statement. Obstet. Gynecol. 2010, 115, $1063-1070$. [CrossRef]

30. Random.org. Available online: http://www.random.org (accessed on 13 November 2018).

31. Galli, M.; De Santis, V.; Tafuro, L. Reliability of the Ahlbäck classification of knee osteoarthritis. Osteoarthr. Cartil. 2003, 11, 580-584. [CrossRef]

32. Weidow, J.; Cederlund, C.-G.; Ranstam, J.; Kärrholm, J. Ahlbäck grading of osteoarthritis of the knee: Poor reproducibility and validity based on visual inspection of the joint. Acta Orthop. 2006, 77, 262-266. [CrossRef]

33. Kesiktas, F.N.; Dernek, B.; Sen, E.I.; Albayrak, H.N.; Aydin, T.; Yildiz, M. Comparison of the short-term results of single-dose intra-articular peptide with hyaluronic acid and platelet-rich plasma injections in knee osteoarthritis: A randomized study. Clin. Rheumatol. 2020, 1-8. [CrossRef] 
34. Ishimoto, Y.; Kawakami, M.; Curtis, E.; Cooper, C.; Harvey, N.; Westbury, L.; Teraguchi, M.; Horie, K.; Nakagawa, Y. The Impact of Lumbar Spinal Stenosis, Knee Osteoarthritis, and Loss of Lumbar Lordosis on the Quality of Life: Findings from the Katsuragi Low Back Pain Study. Spine Surg. Relat. Res. 2018, 3, 157-162. [CrossRef] [PubMed]

35. Marx, F.C.; Oliveira, L.M.; Bellini, C.G.; Ribeiro, M.C.C. Translation and cultural validation of the Lequesne's algofunctional questionnaire for osteoarthritis of knee and hip for portuguese language. Rev. Bras. Reumatol. 2006, 46, 253-260.

36. Sá-Caputo, D.; Paineiras-Domingos, L.L.; Francisca-Santos, A.; Dos Anjos, E.M.; Reis-Silva, A.; Neves, M.F.; Oigman, W.; Oliveira, R.; Brandão, A.A.; Machado, C.B.; et al. Whole-body vibration improves the functional parameters of individuals with metabolic syndrome: An exploratory study. BMC Endocr. Disord. 2019, 19, 6. [CrossRef] [PubMed]

37. Khalil, S.F.; Mohktar, M.S.; Ibrahim, F. The Theory and Fundamentals of Bioimpedance Analysis in Clinical Status Monitoring and Diagnosis of Diseases. Sensors 2014, 14, 10895-10928. [CrossRef]

38. Cômodo AR, O.; Dias AC, F.; Tomaz, B.A.; Silva Filho, A.A.; Werustsky, C.A.; Ribas, D.F.; Spolidoro, J.; Marchini, J.S. Utilização da Bioimpedância Para Avaliação da Massa Corpórea; Associação Médica Brasileira: São Paulo, Brazil, 2009; pp. 1-13.

39. Kyle,U.G.; Bosaeus, I.; De Lorenzo, A.D.; Deurenberg, P.; Elia, M.; Gómez, J.M.; Heitmann, B.L.; Kent-Smith, L.; Melchior, J.-C.; Pirlich, M.; et al. Bioelectrical impedance analysis-part II: Utilization in clinical practice. Clin. Nutr. 2004, 23, 1430-1453. [CrossRef]

40. Kear, B.M.; Guck, T.P.; McGaha, A.L. Timed Up and Go (TUG) Test. J. Prim. Care Community Health 2016, 8, 9-13. [CrossRef]

41. Shariat, A.; Cleland, J.A.; Danaee, M.; Alizadeh, R.; Sangelaji, B.; Kargarfard, M.; Ansari, N.N.; Sepehr, F.H.; Tamrin, S.B.M. Borg CR-10 scale as a new approach to monitoring office exercise training. Work 2018, 60, 549-554. [CrossRef]

42. Neto, S.B.S.; Moreira-Marconi, E.; Kutter, C.R.; Frederico, É.H.F.F.; Paiva, P.D.C.D.; Meyer, P.F.; Chang, S.; Sá-Caputo, D.; Bernardo-Filho, M. Beneficial effects of whole body mechanical vibration alone or combined with auriculotherapy in the pain and in flexion of knee of individuals with knee osteoarthritis. Acupunct. Electro-Ther. Res. 2017, 42, 185-201. [CrossRef]

43. Miot, H.A. Tamanho da amostra em estudos clínicos e experimentais. J. Vasc. Bras. 2011, 10, 275-278. [CrossRef]

44. Trans, T.; Aaboe, J.; Henriksen, M.; Christensen, R.; Bliddal, H.; Lund, H. Effect of whole body vibration exercise on muscle strength and proprioception in females with knee osteoarthritis. Knee 2009, 16, $256-261$. [CrossRef]

45. Bade, M.J.; Kohrt, W.M.; Stevens-Lapsley, J.E. Outcomes before and after total knee arthroplasty compared to healthy adults. J. Orthop. Sports Phys. Ther. 2010, 40, 559-567. [CrossRef]

46. Mizner, R.L.; Petterson, S.C.; Stevens, J.E.; Vandenborne, K.; Snyder-Mackler, L. Early quadriceps strength loss after total knee arthroplasty. The contributions of muscle atrophy and failure of voluntary muscle activation. J. Bone Jt. Surg. Am. 2005, 87, 1047-1053. [CrossRef] [PubMed]

47. Hsiao, Y.-H.; Chien, S.-H.; Tu, H.-P.; Fu, J.C.-M.; Tsai, S.-T.; Chen, Y.-S.; Chen, Y.-J.; Chen, C.-H. Early Post-Operative Intervention of Whole-Body Vibration in Patients After Total Knee Arthroplasty: A Pilot Study. J. Clin. Med. 2019, 8, 1902. [CrossRef] [PubMed]

48. Mikami, Y.; Amano, J.; Kawamura, M.; Nobiro, M.; Kamijyo, Y.; Kawae, T.; Maeda, N.; Hirata, K.; Kimura, H.; Adachi, N. Whole-body vibration enhances effectiveness of "locomotion training" evaluated in healthy young adult women. J. Phys. Ther. Sci. 2019, 31, 895-900. [CrossRef] [PubMed]

49. Coghill, R.C.; Talbot, J.D.; Evans, A.C.; Meyer, E.; Gjedde, A.; Bushnell, M.C.; Duncan, G.H. Distributed processing of pain and vibration by the human brain. J. Neurosci. 1994, 14, 4095-4108. [CrossRef]

50. Zafar, H.; Alghadir, A.; Anwer, S.; Al-Eisa, E. Therapeutic Effects of Whole-Body Vibration Training in Knee Osteoarthritis: A Systematic Review and Meta-Analysis. Arch. Phys. Med. Rehabil. 2015, 96, 1525-1532. [CrossRef]

51. Wang, P.; Yang, X.; Yang, Y.; Yang, L.; Zhou, Y.; Liu, C.; Reinhardt, J.D.; He, C. Effects of whole body vibration on pain, stiffness and physical functions in patients with knee osteoarthritis: A systematic review and meta-analysis. Clin. Rehabil. 2014, 29, 939-951. [CrossRef]

52. Li, X.; Wang, X.Q.; Chen, B.L.; Huang, L.Y.; Liu, Y. Whole-Body Vibration Exercise for Knee Osteoarthritis: A Systematic Review and Meta-Analysis. Evid. Based Complement. Altern. Med. 2015, 2015, 636435. [CrossRef] 
53. Ferreira, R.M.; Duarte, J.A.; Gonçalves, R.S. Non-pharmacological and non-surgical interventions to manage patients with knee osteoarthritis: An umbrella review. Acta Reum. Port. 2018, 43, 182-200.

54. Simão, A.P.; Avelar, N.C.; Tossige-Gomes, R.; Neves, C.D.; Mendonça, V.A.; Miranda, A.S.; Teixeira, M.M.; Teixeira, A.L.; De Andrade, A.G.P.; Coimbra, C.C.; et al. Functional Performance and Inflammatory Cytokines After Squat Exercises and Whole-Body Vibration in Elderly Individuals With Knee Osteoarthritis. Arch. Phys. Med. Rehabil. 2012, 93, 1692-1700. [CrossRef]

55. Salmon, J.R.; Roper, J.A.; Tillman, M.D. Does Acute Whole-Body Vibration Training Improve the Physical Performance of People with Knee Osteoarthritis? J. Strength Cond. Res. 2012, 26, 2983-2989. [CrossRef] [PubMed]

(C) 2020 by the authors. Licensee MDPI, Basel, Switzerland. This article is an open access article distributed under the terms and conditions of the Creative Commons Attribution (CC BY) license (http://creativecommons.org/licenses/by/4.0/). 